\title{
Splenosis Mimicking Recurrence of Renal Cell Carcinoma after Radical Nephrectomy: A Case Report
}

\author{
Joonwhan Kim ${ }^{1}$, Hyosang Hwang ${ }^{1}$, Jun Soo Lee ${ }^{1}$, Joohwan Park', \\ Eun Young Kim ${ }^{2}$, Inkeun Park ${ }^{1}$ \\ Departments of ${ }^{1}$ Internal Medicine and ${ }^{2}$ Radiology, Gachon University Gil Medical Center, \\ Gachon University College of Medicine, Incheon, Korea
}

\begin{abstract}
Splenosis refers to the heterotropic autotransplantation of splenic tissue. Sometimes splenosis after surgical resection is difficult to differentiate from recurrence or metastasis of cancer. A 49-year-old male patient was diagnosed with clear cell renal cell carcinoma of left kidney. As there was no evidence of metastasis, he underwent radical nephrectomy with splenectomy. On surveillance computed tomography, masses at nephrectomy site and pleura were found and both were initially considered to be recurrence. After several cycle of pazopanib administration, pleural mass decreased in size while mass at nephrectomy site did not respond at all. Spleen scan showed increased uptake of the mass and therefore the mass was revealed to be splenosis. To avoid unnecessary treatment and planning optimal treatment, considering the possibility of splenosis is important and spleen scan can be helpful. (Korean J Urol Oncol 2016;14:176-180)
\end{abstract}

Key Words: Splenosis $\cdot$ Accessary spleen $\cdot$ Renal cell carcinoma $\cdot$ Spleen scan $\cdot$ Scintigraphy

Splenosis is the dissemination of splenic cells when the capsule integrity of spleen is breached after trauma or removal of spleen. ${ }^{1}$ It can be found anywhere accessible to the splenic tissue. Reported sites of splenosis after splenectomy are surgical bed of the removed spleen, liver, peritoneal cavity, retroperitoneum, pelvis, and mediastinum. ${ }^{2,3}$ This lesion is sometimes difficult to differentiate from recurrence or metastasis of cancer especially when splenosis develops after curative intent of cancer surgery including splenectomy. ${ }^{4}$ Furthermore, if splenosis or accesory spleen is mistaken for recurrent cancer, patients can undergo unnecessary treatment. ${ }^{5}$

Here we report on a case of a patient with renal cell carcino-

Received June 24, 2016, Revised August 2, 2016,

Accepted August 2, 2016

Corresponding Author: Inkeun Park

Division of Hematology-Oncology, Department of Internal Medicine, Gachon University Gil Medical Center, Gachon University College of Medicine, 21 Namdong-daero 774beon-gil, Namdong-gu, Incheon 21565, Korea

E-mail: ingni79@gilhospital.com

Tel: +82-70-4735-6029, Fax: +82-32-460-1754 ma (RCC) whose accessory spleen on the nephrectomy site was midguided as recurrence of cancer.

\section{CASE REPORT}

A 49-year-old male patient came to the clinic complaining of abdominal discomfort. His computed tomography (CT) scan revealed 6-cm-sized left renal mass involving upper-mid pole which invaded perirenal fat but not not beyond Gerota's fascia. Lymph node enlargement or distant metastasis was not detected (Fig. 1). He was diagnosed with RCC of clinical stage III (cT3aNOM0), and underwent radical nephrectomy in August 2013. Splenectomy was performed together during surgery, because there were a large splenorenal shunt and severe adhesion between spleen and kidney. Pathologically, clear cell type RCC extending to renal sinus and renal vein (pathological stage III, pT3aNOM0) was diagnosed (Fig. 2). Four months after resection, follow-up abdomen-pelvis and chest CT showed a round mass of $2.7-\mathrm{cm}$ size in the nephrectomy surgical bed and

This is an Open Access article distributed under the terms of the Creative Commons Attribution Non-Commercial License (http://creativecommons.org/licenses/by-nc/4.0/) which permits unrestricted non-commercial use, distribution, and reproduction in any medium, provided the original work is properly cited. 2016 (C) Copyright The Korean Urological Oncology Society and The Korean Prostate Society. All Rights Reserved. 
a 2-cm-sized necrotic nodule in the lower lobe of the left lung, and positron emission tomography (PET)-CT also showed a tumorous lesion with increased uptake at the left lower lobe

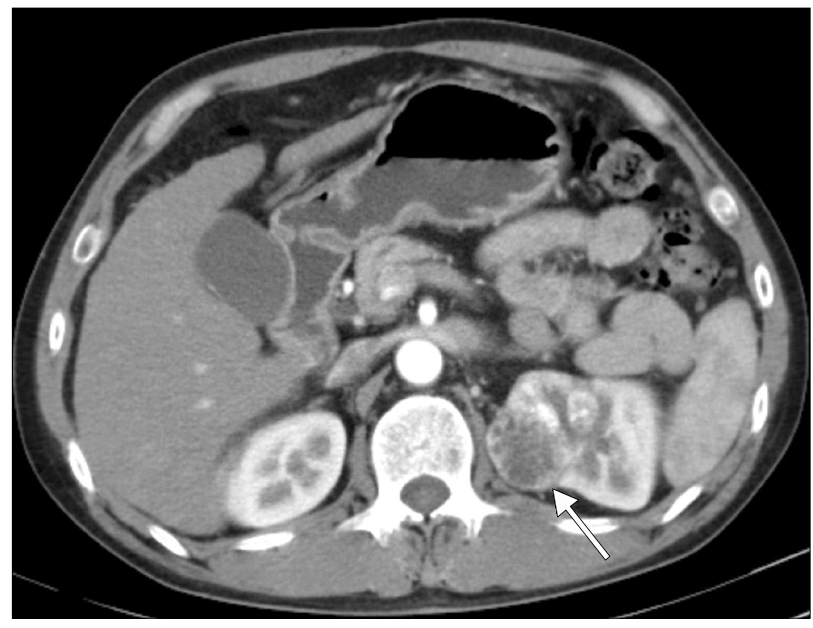

Fig. 1. Computed tomography scan of abdomen demonstrates 6-cm-sized mass involving upper-mid pole of the left kidney, suggesting renal cell carcinoma (arrow).
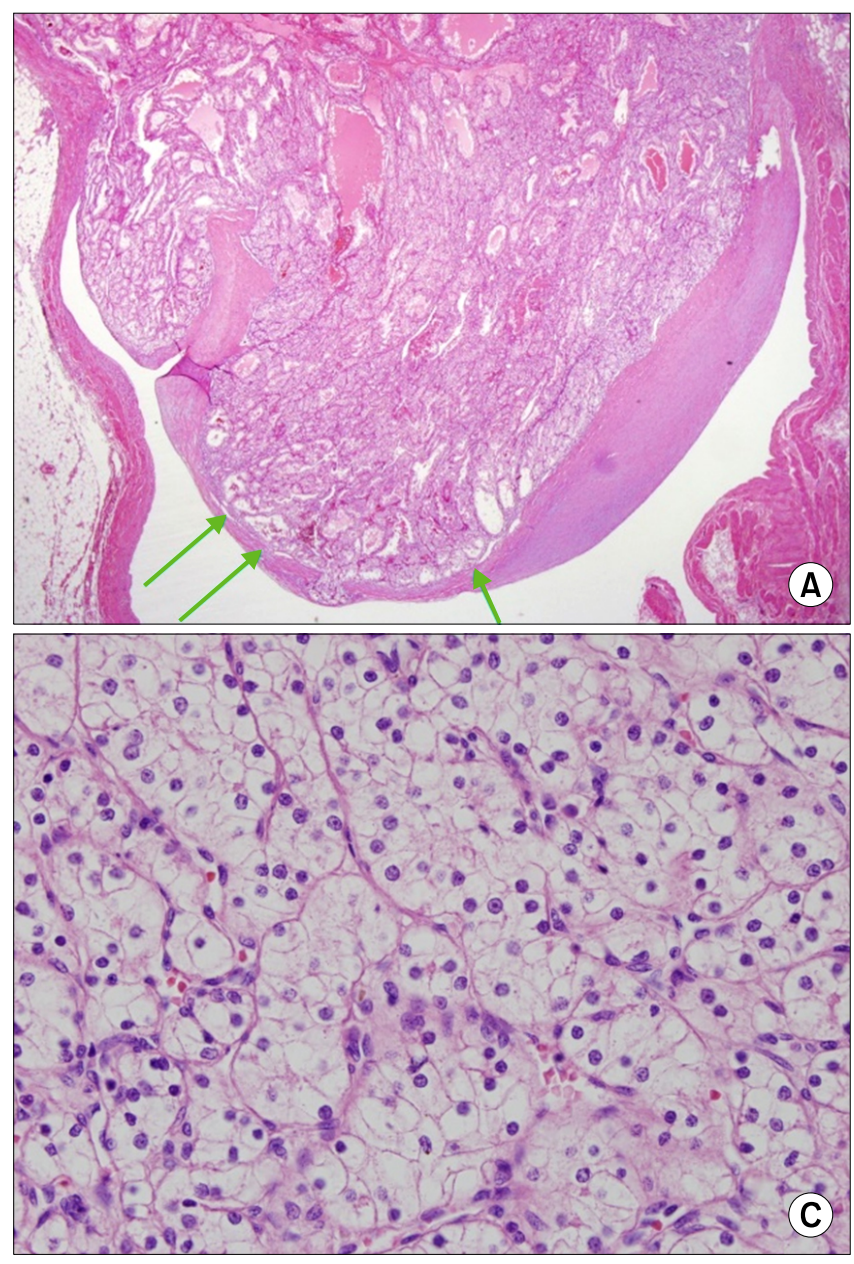

(maximal standardized uptake value $[S U V]=5.40$ ) and the nephrectomy site (maximal SUV=3.26) (Fig. 3). With CT findings and fluorodeoxyglucose (FDG) uptake on PET scan, he was considered to have a recurrent disease at the lung and nephrectomy site and administered $800 \mathrm{mg}$ of pazopanib on December 2013. Because he suffered from grade 2 diarrhea, grade 2 hand foot syndrome, and grade 3 hypertension, the dose of pazopanib was reduced to $600 \mathrm{mg}$ in February 2014.

On CT imaging taken 3 months after pazopanib, the lung lesion had decreased in size $(2.2 \mathrm{~cm} \rightarrow 1.0 \mathrm{~cm})$ while the mass on the nephrectomy site showed no change in size or enhancement. After 6 months after the start of pazopanib, the lung lesion continued to decrease in size while the mass on the nephrectomy site became slightly increased $(2.7 \mathrm{~cm} \rightarrow 3.0 \mathrm{~cm})$ (Fig. 4).

A spleen scan $\left({ }^{99 \mathrm{~m}} \mathrm{Tc}\right.$ heat-damaged red blood cell [RBC] scan) was performed to determine the reason for discordant response between lung and nephrectomy site lesions. A soft tissue lesion at the nephrectomy surgical bed showed increased up-

Fig. 2. Histological examination of kidney showing typical appearance of clear cell type renal cell carcinoma. Renal cell carcinoma extending to renal vein (arrow) (A: H\&E, x12.5; B: H\&E, x200; C: H\&E, x400). 

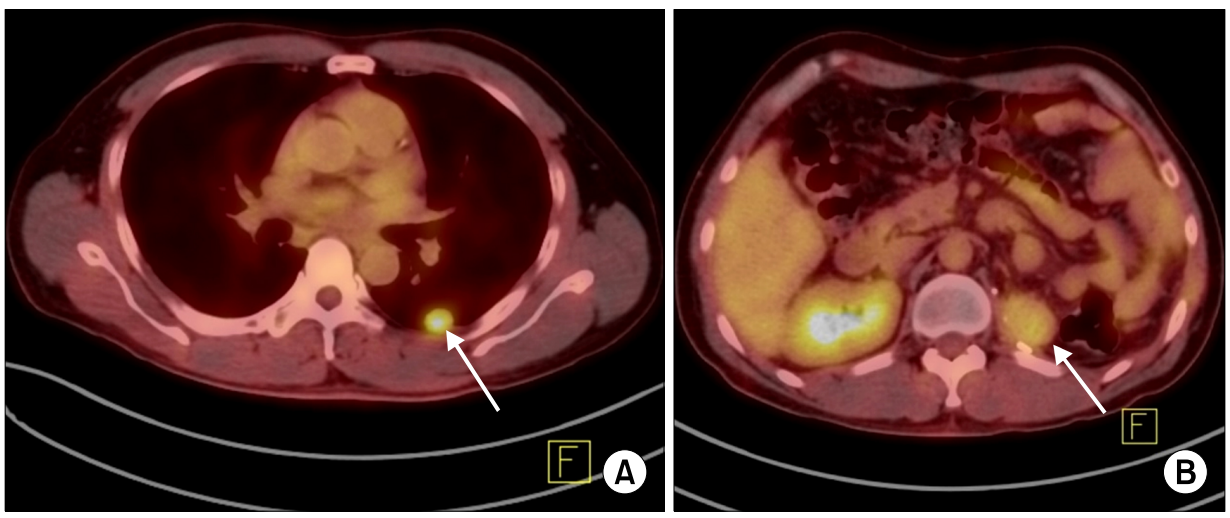

Fig. 3. Torso positron emission tomography-computed tomography scan showed a tumorous lesion with increased uptake at the left lower lobe (arrow) (A: maximal standardized uptake value $[\mathrm{SUV}]=5.40)$ and the nephrectomy site (arrow) (B: maximal SUV=3.26).
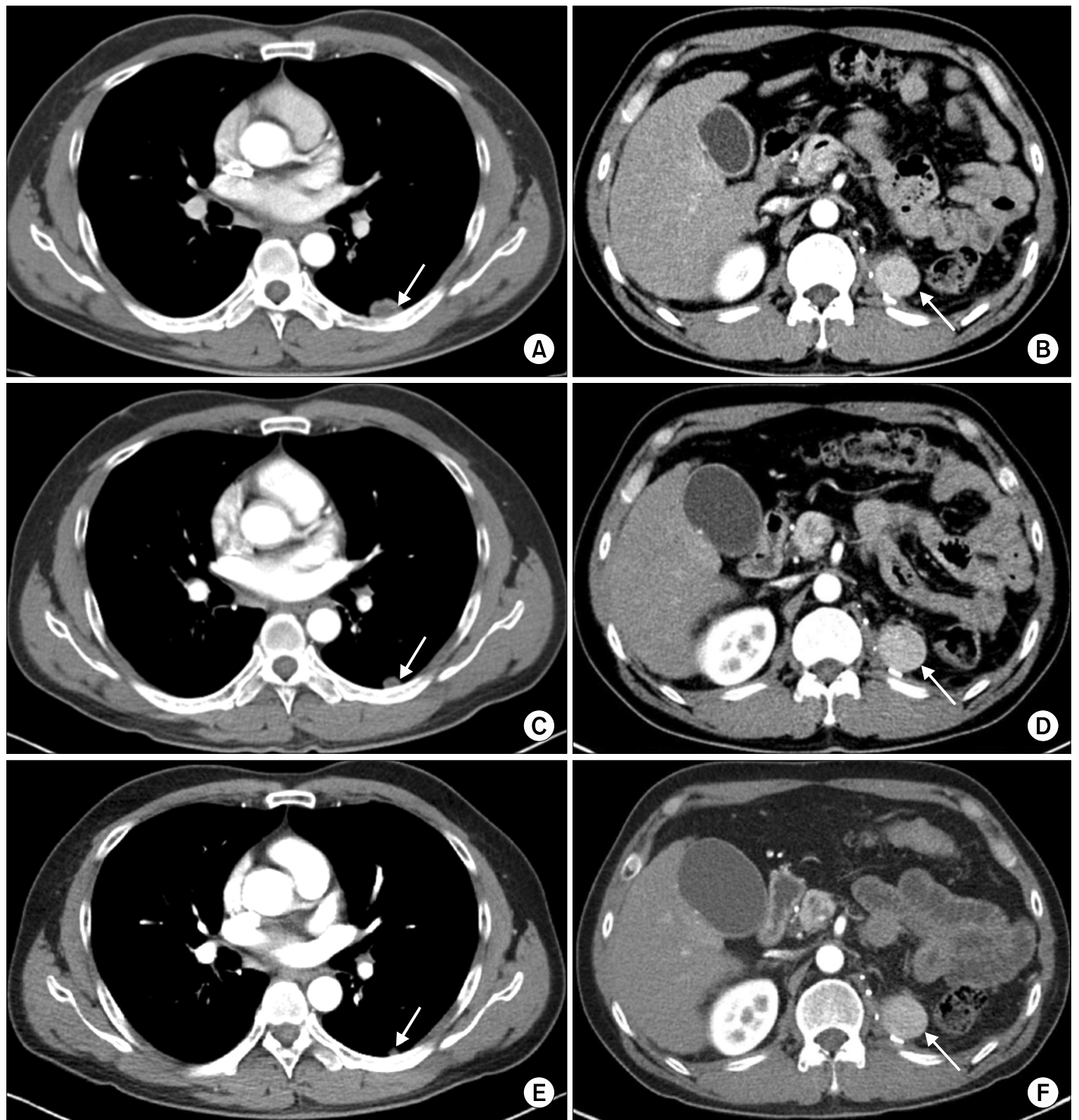

Fig. 4. Consecutive computed tomography scan of chest and abdomen. 4 months after surgery (arrow) (A, B), 3 months after start of pazopanib (arrow) (C, D), and 6 months after pazopanib (arrow) (E, F). 


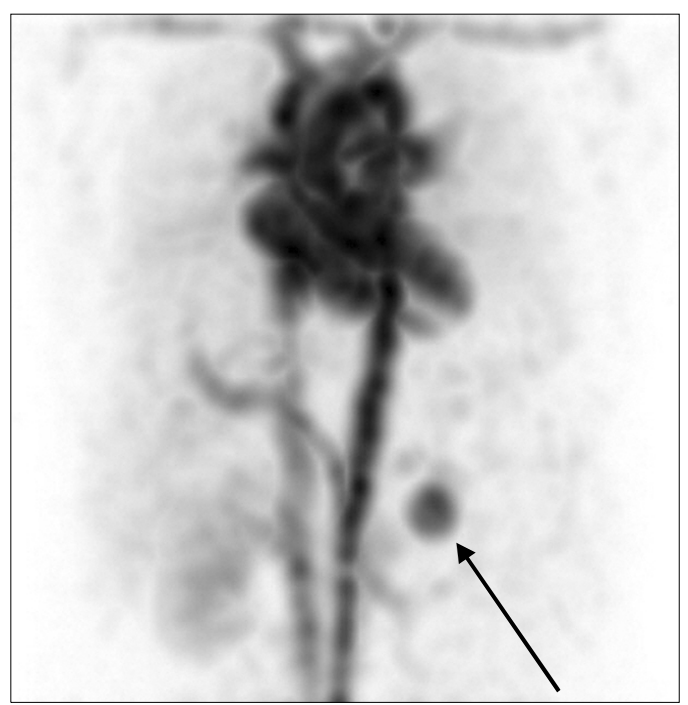

Fig. 5. Spleen scan showed increased uptake in the soft tissue lesion at the left nephrectomy site (arrow), which was found to be splenosis.

take, indicating that the mass was a splenosis (Fig. 5). Because his lung metastasis responded well, pazopanib was continued. $\mathrm{He}$ is alive without disease progression as of January 2016.

\section{DISCUSSION}

Surgical resection can be curative for localized RCC, but, unfortunately, some patients experience recurrence after surgery. If there is any evidence of recurrent or metastatic RCC, administration of systemic treatment with targeted agents (vascular endothelial growth factor tyrosine kinase inhibitors or mammalian target of rapamycin inhibitors) are the current treatment standard. Targeted agents are not without toxicities, and duration of administration is indefinite. Local treatment for metastatic lesions can be a viable option if all of the metastatic lesions are completely resectable and disease progression is not rapid. However, local treatments also entail some risk of complication. ${ }^{6}$

Therefore distinguishing true metastatic disease from its mimics is important for avoiding unnecessary systemic or local treatment. For example, a 69-year-old patient with a history of left-sided RCC underwent radical nephrectomy and subsequent total pancreatectomy with splenectomy for multiple pancreatic metastases 11 years after radical nephrectomy. Twenty months after metastasectomy, follow-up CT showed a new mass suspected as recurrence. Exploratory laparotomy was performed and histological examination of the resected specimen proved to be splenic tissue with no tumorous aspect of the RCC. ${ }^{5}$

In the current case, it was difficult to differentiate recurrence of RCC from splenosis because there was concurrent lung recurrence on CT scan and PET-CT scan showed increased FDG uptake in both lung and splenosis lesions. Among most published cases, splenosis is reported to exhibit normometabolic FDG-PET activity. However, splenosis can present with increased FDG-PET uptake. ${ }^{7}$ Therefore, the extent of FDG-PET uptake cannot be used as an absolute criterion for deciding whether a mass near splenectomy site is a malignant lesion or not. As shown on the spleen scan, the lung lesion was true metastasis while the lesion on the nephrectomy site was not. Splenic scintigraphic scan using ${ }^{99 \mathrm{~m}} \mathrm{Tc}$-sulfur colloid or ${ }^{99 \mathrm{~m}}$ Tc-labeled heat-damaged RBC enable visualization of splenosis or accessory spleen. ${ }^{8}{ }^{99 \mathrm{~m}} \mathrm{Tc}$-sulfur colloid scintigraphy is a popular and easy nuclear tool for the evaluation of splenosis. Yet, colloid accumulating in other lymphatic tissues, especially liver, can result in interpretation errors. Splenic scintigraphy using ${ }^{99 \mathrm{~m}}$ Tc-labeled heat-damaged $\mathrm{RBC}$, which was utilized in our case, provides better specificity and sensitivity in splenectomized patients. Because heat-damaged RBC fragments are larger in size than ${ }^{99 \mathrm{~m}} \mathrm{Tc}$-sulfur colloid materials $(0.3$ to $1.0 \mu \mathrm{m})$, the spleen traps upto $90 \%$ of injected damaged RBCs, whereas it traps only about $10 \%$ of injected Sulfur colloid. ${ }^{9}$ Sensitivity and specificity of splenic scintigraphic scan for detecting splenosis after splenectomy is not known, but referring the study of Ekmekçi et al., ${ }^{10}$ there was no false positive finding in splenic scintigraphy using ${ }^{99 \mathrm{~m}} \mathrm{Tc}$-labeled heat-damaged RBC in the detection of accessory spleens/splenosis in both splenectomized and nonsplenectomized patients. Spleen scan can be economical and very specific in this setting, as previously reported. ${ }^{13}$

We did not consider metastasectomy at initial presentation of recurrence because it appeared to be multiple metastases in separate organs and interval from nephrectomy to recurrence was relatively short (4 months). If we determined that the mass in the nephrectomy surgical bed was accessory spleen, we could strongly consider metastasectomy for the lung lesion instead of systemic treatment which was toxic to the patient.

To avoid futile toxic treatment and for planning of optimal management, awareness of the possibility of accessary spleen is important to both surgical and medical oncologist when a left abdominal mass is discovered after surgery including splenectomy. In addition, spleen scan can be helpful in dis- 
tinguishing splenosis after surgical treatment from recurrence.

\section{CONFLICT OF INTEREST}

The researchers claim no conflicts of interest.

\section{REFERENCES}

1. Varkarakis J, Neururer R, Steiner H, Bartsch G, Peschel R. Splenosis mimicking local recurrence after laparoscopic radical nephrectomy. Urology 2003;62:552

2. Chagnaud C, Champsaur P, Di Costanzo V, Petit P, Chamati $\mathrm{S}$, Charifi $\mathrm{AB}$, et al. Peritoneal splenosis simulating a right retroperitoneal mass. J Radiol 1998;79:1407-9

3. De Riggi MA, Fusco F, Fantini C, D'Agostino A, Cioffi L, Russo G, et al. Laparoscopic surgical treatment of hepatic splenosis. A case report. Ann Ital Chir 2016 Feb 15;87(ePub). pii: S2239253X16024889.

4. Wang G, Chen P, Zong L. Accessory spleen arising from the gastric fundus mimicking gastrointestinal stromal tumor following splenectomy: a case report. Exp Ther Med 2014;7:349-51

5. Tjaden C, Werner J, Buechler MW, Hackert T. Reactive hy- pertrophy of an accessory spleen mimicking tumour recurrence of metastatic renal cell carcinoma. Asian J Surg 2011;34:50-2

6. Yu X, Wang B, Li X, Lin G, Zhang C, Yang Y, et al. The significance of metastasectomy in patients with metastatic renal cell carcinoma in the era of targeted therapy. Biomed Res Int 2015;2015:176373

7. Kellert B, Caster M, Des Jean R, Vaccarello L. Diffuse intra-abdominal splenosis presenting as carcinomatosis exhibiting positron emitted tomography hypermetabolic activity. Gynecol Oncol Case Rep 2013;5:46-8

8. Gunes I, Yilmazlar T, Sarikaya I, Akbunar T, Irgil C. Scintigraphic detection of splenosis: superiority of tomographic selective spleen scintigraphy. Clin Radiol 1994;49:115-7

9. Massey MD, Stevens JS. Residual spleen found on denatured red blood cell scan following negative colloid scans. J Nucl Med 1991;32:2286-7

10. Ekmekçi Ş, Diz-Küçükkaya R, Türkmen C, Adalet I. Selective spleen scintigraphy in the evaluation of accessory spleen/splenosis in splenectomized/nonsplenectomized patients and the contribution of SPECT Imaging. Mol Imaging Radionucl Ther 2015;24:1-7 